DOI: https://doi.org/10.11144/Javeriana.umed58-1.sdas

\title{
Síndrome de disfunción de la articulación sacroiliaca. Análisis clínico de los resultados a corto y mediano plazo de una serie de 115 pacientes tratados con bloqueo selectivo percutáneo
}

\section{Sacroiliac Joint Dysfunction Syndrome. Results of Clinical Analysis in the Short and Medium Term, in a series of 115 Patients Treated with Percutaneous Blockage}

\author{
Laura Abadía ${ }^{1}$ \\ Pontificia Universidad Javeriana, Colombia \\ Juan Carlos Acevedo González ${ }^{2}$ \\ Pontificia Universidad Javeriana, Colombia
}

1 Estudiante duodécimo semestre de Medicina, Pontificia Universidad Javeriana, Bogotá, Colombia

2 Médico neurocirujano especialista en neurocirugía funcional y estereotaxia, manejo de dolor y espasticidad. Director del Departamento de Neurociencias, Hospital Universitario San Ignacio-Pontificia Universidad Javeriana, Bogotá, Colombia.

Cómo citar: Abadía L, Acevedo González JC. Síndrome de disfunción de la articulación sacroiliaca: análisis clínico de los resultados a corto y mediano plazo de una serie de 115 pacientes tratados con bloqueo selectivo percutáneo. Univ Med. 2017;58(1):1-9.

Javeriana.umed58-1.sdas

\section{RESUMEN}

Objetivo: el síndrome de disfunción de la articulación sacroiliaca es una de las principales causas de dolor lumbar. Este artículo muestra cómo se evaluó el curso clínico de personas sometidas a bloqueos selectivos percutáneos para el tratamiento del dolor sacroiliaco. Materiales y métodos: se trataron 115 pacientes que asistieron a consulta entre noviembre de 2006 y mayo de 2013. Fueron diagnosticados con síndrome de disfunción de la articulación sacroiliaca. Se realizaron 131 bloqueos de esta articulación, llevados a cabo por uno de los autores (JCA) con técnica percutánea bajo visión fluoroscópica. El análisis se realizó a partir de la información de la historia clínica con un seguimiento de hasta un año. Resultados: se evaluó la intensidad del dolor con la escala visual análoga y se encontró que el $67 \%$ de los pacientes presentó mejoría superior al $50 \%$ del dolor. El $35 \%$ presentó mejoría superior al $75 \%$ del dolor. Conclusiones: el tratamiento del dolor lumbar secundario a una alteración en la articulación sacroiliaca puede ser tratado con la realización percutánea de bloqueo selectivo con esteroides y anestésicos. Palabras clave

dolor; articulación sacroiliaca; lumbalgia; dolor crónico.

\begin{abstract}
Sacroiliac joint dysfunction syndrome is a major cause of lower back pain. Aim: To discuss the usefulness of selective percutaneous blockages as a potential treatment. Materials and methods: The population of this study were 115 patients who consulted from November 2006 until May 2013, and were diagnosed with sacroiliac joint syndrome. 131 blockages of the sacroiliac joint were performed in the last 6 years by observers (JCA) with a technique under fluoroscopic view. The analysis was performed with data from the clinical history followed for up to one year. Results: It was found that $67 \%$ of patients showed an improvement of over $50 \%$ of pain using analog pain scale of these, 35\% had greater improvement than $75 \%$
\end{abstract}


of the pain. Conclusions: One of the high-cost diseases un the system is lower back pain, and it includes Sacroiliac joint dysfunction Syndrome which must be diagnosed by history physical examination and radiological aids. The percutaneous blockage is a treatment under fluoroscopic vision which can reach very favorable results.

Keywords

pain; low back; chronic pain; sacroiliac joint.

\section{Introducción}

El dolor lumbar es una de las causas más frecuentes de consulta (la segunda causa en Estados Unidos con quince millones de consultas/año), es el quinto motivo de hospitalización y es el tercer motivo de cirugía. Los costos inherentes a su tratamiento son excesivamente altos tanto en los países desarrollados como en el tercer mundo (de cien a doscientos mil millones de dólares en Estados Unidos, relacionados con gastos directos e indirectos). De forma general, el dolor localizado en la región lumbar puede dividirse en aquel que va acompañado de radiculopatía (origen neuropático) y el que no presenta irradiación de características neuropáticas y localización sobre la región lumbar (origen somático) [1,2,3]. El dolor exclusivamente localizado en la región lumbar puede denominarse axial y está asociado más frecuentemente a cambios degenerativos cartilaginosos, articulares, discales y óseos $[4,5,6,7,8,9,10]$. Una de las causas más frecuentes de dolor lumbar axial es la disfunción de la articulación sacroiliaca $[11,12,13,14,15,16]$. Su origen, en un $30 \%$ de los pacientes se origina en las estructuras anatómicas de la articulación sacroiliaca $[3,6,8,15,17,18]$.

Las características del dolor sacroiliaco muestran un componente mecánico (dolor asociado al movimiento) que mejora con el reposo y se puede irradiar por la cara posterior de la pierna sin llegar al pie, de características no neuropáticas [19,20,21,22]. El examen clínico muestra cualquiera de los múltiples signos de provocación sacroiliaca positiva y descarta la presencia de radiculopatía. Y aun cuando la resonancia magnética muestra los cambios degenerativos, es la gammagrafía ósea la que permite identificar el carácter inflamatorio de la articulación. Su tratamiento parte del uso de analgésicos simples (acetaminofén) y antinflamatorios, seguido de procedimientos percutáneos que van desde el bloqueo selectivo con esteroides hasta la termorrizotomía percutánea con radiofrecuencia del ramo dorsal de las raíces espinales sacras $[3,5,7,9,20,23,24,25,26,27]$. El pronóstico es bueno, pero su resultado a largo plazo depende de múltiples factores. Una rehabilitación musculoligamentaria es indispensable para fortalecer el piso pélvico, los músculos de la cintura pélvica y la región lumbar. En este artículo se analiza una serie de pacientes tratados con bloqueo selectivo percutáneo.

\section{Materiales y métodos}

Entre noviembre de 2006 y mayo de 2013, 115 personas consultaron la consulta externa realizada por uno de los autores (JCA). Todos los pacientes tenían una sospecha clínica de síndrome de disfunción de la articulación sacroiliaca a partir de los hallazgos en la anamnesis, el examen clínico y los estudios paraclínicos. La mayoría de los pacientes son de sexo femenino (78 pacientes), que representaba el $68 \%$. Fueron 37 pacientes de sexo masculino (32\%).

$\mathrm{Su}$ distribución, de acuerdo con la edad, mostró que la mayoría se encontraron entre los 40 y los 60 años (58\%). En este grupo hubo una mayor proporción de mujeres (73 $\%)$ con respecto a los hombres (27\%). En el rango de edad que va de los 20 a los 40 años fueron 19 pacientes $(16 \%)$ e, igualmente, fueron mayoría las mujeres (58\%). Los pacientes que se encontraban en el rango de edad 60-80 años fueron el $31 \%$ (61\% de mujeres y $39 \%$ de hombres). Los mayores de 80 años fueron solo el $5 \%$ y se mantuvo la mayor proporción de mujeres (83\% sobre $17 \%$ ).

En todos los pacientes se confirmó el diagnóstico a partir de la presencia de dolor de características mecánicas, uno o varios signos de provocación sacroiliaca y aumento en el índice sacroiliaco $(>1,5)$ en la gammagrafía ósea. 
Se realizaron 131 bloqueos percutáneos de la articulación sacroiliaca (uni o bilateral). Una segunda sesión de bloqueo fue realizada en 16 pacientes.

\section{Técnica}

Cada paciente se ubicó en una posición de decúbito prono con un rollo de tela colocado sobre las espinas iliacas anterosuperiores para levantar el hueso sacro, mientras que sus articulaciones se pusieron en el cenit. Se realizó asepsia y antisepsia de la región lumbosacra. Bajo visión fluoroscópica se identificó el tercio inferior de la articulación sacroiliaca ("verdadera articulación”) y el espacio óseo ubicado entre una línea trazada sobre el borde lateral de los forámenes sacros y la articulación sacroiliaca. Se realizó punción con aguja en un punto en cada articulación sacroiliaca y se aplicaron $2 \mathrm{~cm} 3$ de roxicaína sin epinefrina al $1 \%$. Se realizó punción con Spinocan n. 25, dirigido hasta el espacio intrarticular, donde se aplicaron $5 \mathrm{~cm} 3$ de una mezcla de lidocaína al $1 \%$ sin epinefrina y dexametasona $(6 \mathrm{~cm} 3+4 \mathrm{~cm} 3)$. La aguja se orientó hasta el espacio atravesado por los ramos dorsales de las raíces espinales sacras, donde se aplicaron los $5 \mathrm{~cm} 3$ de la mezcla descrita. El procedimiento es ambulatorio. Se indicó reposo al paciente durante 48 horas y luego reinicio de las actividades.

\section{Seguimiento}

Todos los pacientes fueron evaluados en consulta externa una semana después del procedimiento. A noventa pacientes se les llamó telefónicamente para evaluar el resultado del procedimiento a largo plazo. Este seguimiento se hizo en todos los pacientes con una duración de entre nueve meses y cinco años; así mismo, se les evaluó la intensidad del dolor durante su tratamiento con la Escala Visual Análoga.

\section{Resultados}

Se evaluó la mejoría clínica con la Escala Visual Análoga y se clasificaron los resultados en cinco grupos de acuerdo con el porcentaje de mejoría clínica. Grupo 1: mejoría del $75 \%$ al $100 \%$; grupo 2: mejoría de entre el $50 \%$ y el $74 \%$; grupo 3: mejoría de entre el $25 \%$ y el $49 \%$; grupo 4: mejoría inferior al $25 \%$, y grupo 5: ausencia completa de mejoría clínica. Los hallazgos en cada uno de los grupos son los siguientes:

Grupo 1: fueron un total de 40 pacientes (35 $\%$ del total) quienes presentaron una mejoría superior al $75 \%$ en la valoración inicial y persistió hasta el último control. Fueron 26 mujeres (65 $\%)$ y 14 hombres (35\%). Identificamos en este grupo de pacientes que el $27 \%$ de ellos, siendo mujeres, eran multíparas. La frecuencia de cirugía de columna previa fue alta. Fueron 11 pacientes quienes habían tenido un tratamiento quirúrgico de la columna lumbar (27\%). El $10 \%$ de los pacientes tenía enfermedades autoinmunes.

Grupo 2: fueron 37 pacientes (32\%). La mayoría de ellos fueron mujeres $(24$ pacientes $=$ $65 \%)$. Los hombres eran 13 (35\%).

Grupo 3: fueron 20 pacientes (17\%). Fueron el $77 \%$ de mujeres y el $23 \%$ de hombres. Un 35 $\%$ de los pacientes (mujeres) eran multíparas.

Grupo 4: fueron 5 pacientes (4\%). En este grupo, el $40 \%$ de ellos había sido sometido a tratamiento quirúrgico de la columna lumbar. Todos eran mujeres.

Grupo 5: fueron 13 pacientes (11\%).

\section{Discusión}

El dolor lumbar es uno de los síntomas más frecuentes en la población general. Su incidencia y prevalencia muestra que hace parte de las patologías que afectan económicamente con más fuerza de forma directa e indirecta la salud pública de los países desarrollados y, por supuesto, de los menos desarrollados. Los gastos inherentes a su tratamiento y los relacionados con pérdida de capacidad laboral, incapacidades y alteración en la calidad de vida son excesivamente altos. En Estados Unidos se calcula que son 
superiores a 61.000 millones de dólares anuales $[28,29,30,31,32,33,34,35,36,37,38,39,40] . \quad$ En 1998, Waddel determinó cómo solo el $15 \%$ de los pacientes con dolor lumbar podía tener un diagnóstico preciso del elemento estructural causante del síntoma. El mejor conocimiento de la región lumbar y, por supuesto, de los fenómenos dolorosos localizados en ella es relevante para realizar un tratamiento adecuado y un gasto razonable de los recursos médicos.

La articulación sacroiliaca es una de las estructuras de mayor importancia en esta región. Cumple un papel biomecánico básico que permite una adecuada absorción y distribución de las fuerzas biomecánicas, pues protege estructuras anatómicas más sensibles a este tipo de esfuerzo. Distribuye y compensa los esfuerzos biomecánicos de la columna vertebral, los miembros inferiores y la pelvis $[1,4,5,8,11,14,17,23,31,34,41,42,43,44,45,46,47,48,49,50]$. Aunque desde comienzos del siglo pasado se describió la articulación sacroiliaca como una estructura esencial en el funcionamiento normal de la región lumbar, el desconocimiento de muchos de sus elementos semiológicos y fisiológicos es grande. La articulación sacroiliaca puede ser la causa del dolor en un $15 \%-30 \%$ de los pacientes con patología lumbosacrococcígea y es la causante de dolor glúteo en el $50 \%$ de los pacientes deportistas [6,8-11,28,29,51-64]

La articulación sacroiliaca es una estructura relevante en el funcionamiento biomecánico humano. Cumple una doble función al ser la porción terminal de la columna vertebral y el cierre posterior de la cavidad pélvica. En la columna vertebral, su función es comunicar los esfuerzos biomecánicos a la pelvis y a las extremidades inferiores mediante las articulaciones de la cadera $[2,9,11,17,35,44,47,65-76]$.

En la cavidad pélvica, tiene una función de cierre posterior y de protección de las estructuras intrapélvicas. La cintura pélvica alberga y protege las vísceras y el feto durante el embarazo. Igualmente, la pelvis tiene la capacidad de distenderse para permitir el paso del feto a través del canal vaginal
$[19,35,28,72,77,78,79,80,81,82]$. Durante el parto, la articulación sacroiliaca, por contener una gran cantidad de ligamentos, logra arcos de movimiento mínimos; pero suficientes para permitir el aumento del diámetro de la cintura pélvica $[1,5,47,72,74,83,84]$.

La cavidad pélvica se compara con una bóveda donde la cerradura es el hueso sacro. Es la cerradura estructural; pero igualmente funcional. El sacro permite abrir o cerrar esa bóveda de acuerdo con su movimiento o su desplazamiento (nutación o contranutación). Con los movimientos del tronco y de las extremidades, hay un desplazamiento inmediato de fuerzas a lo largo de la pelvis. En la posición de pie, aparecen dos vectores de fuerza que, de la columna vertebral, se dirigen a las cabezas femorales pasando por la articulación sacroiliaca y los huesos iliacos. En la posición de pie, el hueso sacro se impacta en la pelvis, entre los dos huesos iliacos, y producen un efecto de cierre con aducción de los alerones iliacos. Este movimiento genera un mecanismo de protección de la pelvis que absorbe parte de esos vectores de fuerza que van a proyectarse a las cabezas femorales.

La relación biomecánica entre la articulación coxofemoral y la articulación sacroiliaca es directa. En condiciones normales, la articulación coxofemoral envía sus vectores de fuerza a las extremidades que se encargan de distribuirlos y aboserberlos pero si en las extremidades hay rigidez o alguna limitación para realizar esa función, la trasmisión se hace directamente a la articulación sacroiliaca $[28,82,85]$.

La estructura de la articulación sacroiliaca permite corroborar su importancia biomecánica. En el plano frontal, las carillas articulares son oblicuas hacia abajo y adentro, y favorecen la impactación del sacro sobre los dos huesos iliacos. En el plano horizontal, el sacro tiene tendencia a desplazarse hacia adelante que favorece el mecanismo de encastramiento que acerca los alerones iliacos y compacta los huesos de la pelvis. Esa disposición del sacro tiene un doble mecanismo autobloqueante en el que entre más fuerza se ejerce sobre el sacro, mayor es el mecanismo de empotramiento del sacro sobre los iliacos y mayor la tensión sobre los 
ligamentos. Este mecanismo permite disminuir el impacto directo en los discos intervertebrales, amortigua vibraciones y rotaciones. Igualmente, la distribución actual del sacro entre los iliacos es una estructura que permitió en el desarrollo filogenético la posición de pie del humano.

En ese contexto, cuando se presenta una lumbarización de la vértebra S1, la función descrita no se puede realizar adecuadamente y aparece el dolor. También el hecho de que la articulación tenga unas grandes carillas articulares planas permite que, a través de ellas, se transmitan grandes fuerzas biomecánicas, pero igualmente hacen de ellas más sensibles y menos resistentes, sobre todo para aquellos movimientos de cizalla. La articulación suple esa dificultad ósea con la presencia de fuertes cartílagos [29,30,63,71,86].

La articulación sacroiliaca es una estructura compleja. En ella es múltiple el origen del dolor. Se incluyen los desgarros de la cápsula, la ruptura de la sinovial, la distensión ligamentaria, las alteraciones en la movilidad, estrés biomecánico, microfracturas espontáneas o traumáticas, disfunción musculoligamentaria, entre otras. Las patologías de la articulación sacroiliaca que producen dolor pueden clasificarse en dos grandes grupos asociados o no a alteraciones reumatológicas. Esta división diferencia las patologías aisladas de la región sacroiliaca (grupo 1) de aquellas que se asocian a patologías reumatológicas sistémicas (grupo 2). Esta clasificación está asociada al pronóstico terapéutico. Al ser el grupo 1 una patología focalizada, la posibilidad de una adecuada respuesta a las opciones terapéuticas es mayor. El grupo 2, al estar asociado a una afectación sistémica, tiene mayor posibilidad de convertirse en una patología crónica con baja respuesta a los tratamientos específicos [17,29,30,63,72,81].

Los estudios paraclínicos son de gran utilidad en el diagnóstico y tratamiento del dolor sacroiliaco; no solo nos permiten confirmar o descartar la presencia de una enfermedad reumatológica asociada, sino que confirman que el origen del dolor es articular, óseo, muscular o ligamentario. Aunque hay muchos elementos clínicos que ayudan a confirmar el diagnóstico de afectación de la articulación sacroiliaca, muchos no son conocidos por los especialistas. Además, seguimos expuestos al "mito" erróneo que asocia todo dolor de la región lumbar y glútea a la presencia de una hernia discal. Por eso, los estudios paraclínicos ayudan a corroborar la sospecha clínica de alteración en la articulación sacroiliaca. La gammagrafía ósea es un examen de gran importancia en el diagnóstico de patologías inflamatorias y degenerativas (activas), aun cuando se considera que tiene una gran especificidad diagnóstica $(90$ $\%)$ y con una muy baja sensibilidad (13-40 \%).

El tratamiento del síndrome de disfunción de la articulación sacroiliaca incluye el uso de analgésicos comunes, antinflamatorios (en sus fases agudas) y rehabilitación física orientada al fortalecimiento de las estructuras musculoligamentarias. El papel del bloqueo selectivo de la articulación sacroiliaca tiene una doble función, tanto diagnóstica como terapéutica. La mejoría del dolor por un periodo superior a veinticuatro horas puede ser la confirmación de la sospecha diagnóstica del síndrome. Sin embargo, como lo muestran los resultados de este artículo, su utilidad no se limita a una confirmación diagnóstica, sino que permite un control adecuado del dolor a largo plazo, un inicio rápido del proceso de rehabilitación y, por supuesto, reintegro social y laboral.

\section{Conclusiones}

El bloqueo selectivo de la articulación sacroiliaca es un procedimiento útil en el tratamiento del dolor lumbar.

\section{Referencias}

1. Acevedo JC. Dolor lumbar crónico. El Informador Médico. 2002;85:24-6.

2. Acevedo JC. Síndrome facetario lumbar: nuevo signo de diagnóstico clínico. Revista Española de Medicina Física y Rehabilitación. 2004;38(4):168-75.

3. Acevedo JC. Esteroides epidurales lumbares: análisis clínico de una serie de pacientes con 
dolor lumbar y radiculopatía. Actualización en Neurocirugía. 2006;3 (1):15-23.

4. Acevedo JC. Síndrome facetario: revisión de la experiencia clínica en el Hospital San Ignacio. Neurociencias en Colombia. 2006;14(3):111-24.

5. Acevedo JC. Dolor lumbar discogénico: tratamiento intradiscal con radiofrecuencia. Presentación de caso clínico. Seguimiento a 8 meses. Dolor. 2007;2(1):69-82.

6. Acevedo JC. Síndrome facetario: revisión de la evidencia clínica y experiencia en el Hospital Universitario de San Ignacio. Dolor. 2007;2(2):49-60.

7. Weksler N, Velan GJ, Semionov M. The role of sacroiliac joint dysfunction in the genesis of low back pain: The obvious is not always right. Arch Orthop Trauma Surg. 2007;127:885-8.

8. Bogduk N. Low back pain. En: Clinical anatomy of the lumbar spine and sacrum. 4th ed. Sydney: Elsevier; 2005. p. 183-216.

9. Laslett M, Oberg B, Aprill CN, McDonald B. Zygapophysial joint blocks in chronic low back pain: a test of Revel 's model as a screening test. BMC Musculoskelet Dis. 2005 Nov 16;5:43. doi: 10.1186/1471-2474-5-43

10. Sembrano JN, Polly DW. How often is low back pain not coming from the back? Spine. 2008;34(1): E27-32.

11. Schwarzer AS, Aprill CN, Bogduk N. The sacroiliac joint in chronic low back pain. Spine. 1995;20(1):31-7.

12. Maige JY, Planchon CA. Sacroiliac joint pain after lumbar fusion: A study with anesthetic blocks. Eur Spine J. 2006;15(1):8-15.

13. Dreyfudd P, Dreyer S, Cole A, et al. Sacroiliac joint pain. J Am Acad Orthop Surg. 2004;12:255-65.

14. Bernard TN, Cassidi JD. The sacroiliac syndrome: Pathophysiology, diagnosis, and management. En: Frymoyer JW, editor. The adult spine: Principles and practice. New York : Raven; 1991. p. 2107-30.

15. Yamashita T, Minaki Y, Oota I, et al. Mechanosensitive afferent units in the lumbar intervertebral disc and adjacent muscle. Spine. 1993;18:2252-6.

16. Minaki Y, Yamashita $\mathrm{T}$, Ishii $\mathrm{S}$. An electrophysiological study on the mechanoreceptors in the lumbar spine and adjacent tissues. Neurol Orthop. 1996;20:23-35.

17. Ebraheim Na, Lu J, Biyani A, et al. The relationship of lumbosacral plexus to the sacrum and the sacroiliac joint. Am J Orthop. 1997;26:105-10.

18. Brunner C, Kissling R, Jacob HA. The effects of morphology and histopathologic findings on the mobility of the sacroiliac joint. Spine. 1991;16:1111-7.

19. Vrahas $M$, hern TC, Diangelo $D$, et al. Ligamentous contributions to pelvic stability. Orthopedics. 1995;18:271-4.

20. O'Seha FD, Boyle E, Salonen DC, et al. Inflammatory and degnerative sacroiliac joint in a primary back pain cohort. Arthritis Care Res. 2010;62(4):447-54.

21. Chou LH, Slipman CW, Bhagia SM, et al. Inciting events initiating injectionproven sacroiliac joint syndrome. Pain Med. 2004;5:26-32.

22. Pohlemann T, Tscherne H, Baumgartel F, et al. Pelvic fractures: epidemiology, therapy and long-term outcome. Overview of the multicenter study of the Pelvis Study Group [in German]. Unfallchirrg. 1996;99:160-7.

23. Vanelderen P, Szadek K, Cohen SP, et al. 13. Sacroiliac joint pain. Pain Pract. 2010;10(5):470-8. doi: 10.1111/ j.1533-2500.2010.00394.x

24. Dreyfuss P, Michaelsen M, Pauza K, et al. The value of medical history and physical examination in diagnosing sacroiliac joint pain. Spine. 1996;21:2594-2602.

25. Slipman CW, Jackson HB, Liptez JS, et al. Sacroiliac joint pain referral zone. Arch Phys Med Rehabil. 2000;81:334-8.

26. Szadek KM, van der Wurff P, van Tulder MW, et al. Diagnostic validity of criteria for sacroiliac joint pain: a systematic review. J Pain. 2009;10:354-68.

27. Fortin JD, Dwyer AP, West S, et al. Sacroiliac joint: pain referral maps upon applying 
a new injection/arthrography technique. Spine. 1994;19:1475-82.

28. Maigne JY, Boulahdour H, Chatellier G. Value of quantitative radionuclide bone scanning in the diagnosis of sacroiliac joint syndrome in 32 patients with low back pain. Eur Spine J. 1998;7:328-31.

29. Manchikanti I, Pampati V, Fellows B. The inability of the clinical picture to characterize pain from facet joint. Pain Physician. 2009;3:158-66.

30. McKenzie RA, May S. The lumbar spine: Mechanical diagnosis and therapy. 2nd ed. Waikanae: Spinal Publication; 2003.

31. Bernard TN, Kirkaldy-Willis H. Recognizing specific characteristics of nonspecific low back pain. Clin Orthop Rel Res. 1978;217:266-80.

32. Ha K, Lee S, Kim K. Degeneration of sacroiliac joint after instrumented lumbar or lumbosacral fusion. Spine. 2008;33(11):1192-8.

33. Slipman CW, Sterenfeld EB, Chou LH, et al. The value of radionuclide imaging in the diagnosis of sacroiliac joint syndrome. Spine. 1996;21:2251-4.

34. Braun J, Sieper J, Bollow B. Imaging of sacroiliitis. Clin Rheumatol. 2000;19:51-7.

35. Rosenberg JM, Quint DJ, de Rosayro AM. Computerized tomographic localization of clinically-guided sacroiliac joint injections. Clin J Pain. 2000;16:18-21.

36. Pulisetti D, Ebraheim NA. CT-guide sacroiliac joint injections. J Spinal Disord. 1999;12(4):310-312.

37. Ivanov AA, Kiapour A, Ebraheim NA, et al. Lumbar fusion leads to increases in angular motion and stress across sacroiliac joint. Spine. 2009;34(5):E162-9.

38. Boswell MV, Trescot AM, Datta D, et al. Interventional techniques: evidence-based practice guidelines in the management of chronic spinal pain. Pain Phys. 2007;10(1):7-111.

39. Buchowski JM, Kebaish KM, Sinkov V, et al. Functional and radiographic outcome of sacroiliac arthrodesis for the disorders of the sacroiliac joint. Spine J. 2005;5(5):520-8.
40. Schütz U, Grob D. Poor outcome following bilateral sacroiliac joint fusion for degenerative sacroiliac joint syndrome. Acta Orthop Belg. 2006;72(3):296-308.

41. Vanelderen P, Szadek K, Cohen SP, et al. Sacroiliac joint pain. En: Evidence based interventional pain medicine: According to clinical diagnoses. Philadelphia: John Wiley \& Sons; 2012. p. 96-102. doi: 10.1002/9781119968375.ch13

42. Gevargez A, Groenemeyer D, Schirp S, Braun M. CT-guided percutaneous radiofrequency denervation of the sacroiliac joint. Eur Radiol. 2002;13:1360-5.

43. Ferrante FM, King LF, Roche EA, et al. Radiofrequency sacroiliac joint denervation of sacroiliac syndrome. Reg Anesth Pain Med. 2001;26:137-42.

44. Cohen SP, Abdi S. Lateral branch blocks as a treatment for sacroiliac joint pain: A pilot study. Reg Anesth Pain Med. 2003;28:113-9.

45. Vallejo R, Benyamin RM, Kramer J, Stanton G, Joseph NJ. Pulsed radiofrequency denervation for the treatment of sacroiliac joint syndrome. Pain Med. 2006;7:429-34.

46. Cosman ER Jr, Gonzalez CD. Bipolar radiofrequency lesion geometry; implications for palisade treatment of sacroiliac joint pain. Pain Pract. 2011;2(1):18-34.

47. Laslett M, Aprill CN, McDonald B, Young SB. Diagnosis of sacroiliac joint pain: Validity of individual provocation test and composites of tests. Man Ther. 2005;10:207-18.

48. Van der Wurff P, Buijs EJ, Groen GJ. A multitest regimen of pain provocation tests as an aid to reduce unnecessary minimally invasive sacroiliac joint procedures. Arch Phys Med Rehabil. 2006;87:10-4.

49. Dreyfuss P, Snyder BD, Park K, Willard F, Carreiro J, Bogduk N. The ability of single site, single depth sacral lateral branch blocks to anesthetize the sacroiliac joint complex. Pain Med. 2008;9:844-50.

50. Cohen SP, Hurley RW, Buckenmaier CC 3rd, Kurihara C, Morlando B, Dragovich A. Randomized placebo controlled study 
evaluating lateral branch radiofrequency denervation for sacroiliac joint pain. Anesthesiology. 2008;109:279-88.

51. Szadek KM, Hoogland PV, Zuurmond WW, de Lange JJ, Perez RS. Nociceptive nerve fibers in the sacroiliac joint in humans. Reg Anesth Pain Med. 2008;33:36-43.

52. Solonen KA. The sacroiliac joint in the light of anatomical, roentgenological and clinical studies. Acta Orthop Scand Suppl. 1957;27:1-127.

53. Dreyfuss P, Henning T, Malladi N, Goldstein B, Bogduk N. The ability of multi-site, multi-depth sacral lateral branch blocks to anesthetize the sacroiliac joint complex. Pain Med. 2009;10:679-88.

54. Cohen SP, Strassels SA, Kurihara C, et al. Outcome predictors for sacroiliac joint (lateral branch) radiofrequency denervation. Reg Anesth Pain Med. 2009;34:206-14.

55. McGrath MC, Zhang M. Lateral branches of dorsal sacral nerve plexus and the long posterior sacroiliac ligament. Surg Radiol Anat. 2005;27:327-30.

56. McDougall JJ. Arthritis and pain. Neurogenic origin of joint pain. Arthritis Res Ther. 2006;8:220.

57. Grob KR, Neuhuber WL, Kissling RO. Innervaction of the sacroiliac joint of the human [in German]. Z Rheumatol. 1995; 54:117-22.

58. Vilensky JA. O'Connor BL, Fortin JD, Merkel GJ, Jimenez AM, Scofield BA, Kleiner JB. Histologic analysis of neural elements in the human sacroiliac joint. Spine. 2002;27:1202-7.

59. Carrera GF Lumbar facet joint injection back pain and sciatica: Preliminary results Radiology J. 1980;137:665-7.

60. Drezner JA, Herring SA. Managing lowback pain. Steps to optimize function and hasten return to activity. Phys Sport Med. 2001;29:37-43.

61. Deyo RA, Cherkin D, Conrad D, et al. Cost, controversy, crisis: low back pain and the health of the public. Ann Rev Public Health. 1991;12:141-56.
62. Deville WL, Buntinx F, Bouter LM. Conducting systematic reviews of diagnostic studies: didactic guidelines. BMC Med Res Methodol. 2002;2:9.

63. Manchikanti L, Pamparti V, Fellows B. Prevalence of lumbar facet joint pain in chronic low back pain. Pain Physician. 1999;2:59-64.

64. Fortin JD, Kissling RO, O'Connor BL, Vilensky JA. Sacroiliac joint innervations and pain. Am J Orthop. 1999;28:687-90.

65. Murata Y, Takahashi K, Ohtori S, Moriya $\mathrm{H}$. Innervation of the sacroiliac joint in rats by calcitonin gene-related peptide-immunoreactive nerve fibers and dorsal root ganglion neurons. Clin Anat. 2006;20:82-8.

66. Fortin JD, Washington MJ. Three pathways between the sacroiliac joint and neural structures ANJR Am J Neuroradiol. 1999;20:1429-34

67. Yin W, Willard F, Carreiro J, Dreyfuss P. Sensory stimulation-guided sacroiliac joint radiofrequency neurotomy: Technique based on neuroanatomy of the dorsal sacral plexus. Spine. 2003 Oct 15;28(20):2419-25. doi: 10.1097/01.BRS.0000085360.03758.C3

68. Fortin JD, Vilensky JA, Merkel GJ. Can the sacroiliac joint cause sciatica? Pain Phys. 2003;6:269271.

69. Jaovisidha S, Ryu KN, De Maeseneer M, Haghighi P, Goodwin D, Sartoris DJ, Resnick D. Ventral sacroiliac ligament: Anatomic and pathologic considerations. Invest Radiol. 1996;31:532-41.

70. McLain RF. Mechanoreceptor endings in human cervical facet joints. Spine. 1994;19:495-501.

71. Cohen SP. Sacroiliac joint pain: A comprehensive review of anatomy, diagnosis, and treatment. Anesth Analg. 2005;101:1440-53.

72. Airaksinen O, Brox JI, Cedraschi C. Chapter 4. European guidelines for the management of chronic nonspecific low back pain. Eur Spine J. 2006;15(Suppl 2):S192-S300. 
73. Young A, April C, Laslett M. Correlation of clinical examination characteristics three sources of chronic low back pain. Spine J. 2003;3:460-5.

74. Merskey H, Bogduk N. Classification of chronic pain: Description of chronic pain syndromes and definitions of pain terms. 2nd ed. Seattle: International Association for the Study of Pain; 1994.

75. Derby R, Kim BJ, Lee SH. Comparison of discographic findings in asymptomatic subject discs and the negative discs of chronic LBP patients: Can discography distinguish asymptomatic discs among morphologically abnormal discs? Spine J. 2005;5:389-94.

76. Bechtel R. Physical characteristics of the axial in Terosseous ligament of the human sacroiliac joint. Spine J. 2001;1:255-9.

77. Bakland O, Hansen JH. The axial sacroiliac joint. Anat Clin. 1984;6:29-36.

78. Gibson SJ, Farrell MA. Review of age differences in the neurophysiology of nociception and the perceptual experience of pain. Clin J Pain. 2004;20:227-39.

79. Coppers MH, Marani E, Thomeer RT, Groen GJ. Innervation of "painful" lumbar discs. Spine. 1997;22:2342-9.

80. Brown MF, Hukkanen MV, McCarthy ID, Redfern Dr, Batten JJ, Crock HV, Hughes SP, Polak JM. Sensory and sympathetic innervation of the vertebral endplate in patients with degenerative disc disease. J Bone Joint Surg Br. 1997;79:147-53.

81. Hansen HC, McKenzie-Brown AM, Cohen SP, Swicegood JR, Colson JD, Manchikanti L. Sacroiliac join interventions: A systematic review. Pan Physician. 2007;10(1):165-84.

82. Laslett M, McDonald B, Aprill CN. Clinical predictors of screening lumbar zygapophysial joint blocks: Development of clinical prediction rules. Spine J. 2006;6:370-9.

83. Laslett M, Oberg B, Aprill CN. Centralization as a predictor of provocation discography results in chronic low back pain, and influence of disability and distress on diagnostic power. Spine J. 2005;5:370-80.

84. Laslett M, Oberg B, Aprill CN. A study of clinical predictors of lumbar discogenic pain as determined by provocation discography. Eur Spine J. 2006;15:1473-84.

85. Lim CH, Jee W-H, Son BC. Discogenic lumbar pain association with MR imaging and CT discography. Eur J Radiol. 2005;54:431-7.

86. Bogduk N. The anatomical basis for spinal pain syndromes. J Manipulative Physiol Ther. 1995;18:603-5. 